\title{
Photo-dissociation of naphthalene dimer cations stored in a compact electrostatic ion storage ring
}

Cite as: J. Chem. Phys. 150, 054303 (2019); https://doi.org/10.1063/1.5055939

Submitted: 12 September 2018 . Accepted: 14 January 2019 . Published Online: 07 February 2019

J. Bernard (D), A. Al-Mogeeth, A.-R. Allouche (D), L. Chen, G. Montagne, and S. Martin

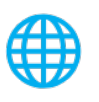

\section{ARTICLES YOU MAY BE INTERESTED IN}

Vacuum ultraviolet excited state dynamics of small amides

The Journal of Chemical Physics 150, 054301 (2019); https://doi.org/10.1063/1.5079721

The effect of Coulomb repulsion on the space-time resolution limits for ultrafast electron diffraction

The Journal of Chemical Physics 150, 054201 (2019); https://doi.org/10.1063/1.5060673

On the choice of coordinates in anharmonic theoretical vibrational spectroscopy: Harmonic vs. anharmonic coupling in vibrational configuration interaction

The Journal of Chemical Physics 150, 054107 (2019); https://doi.org/10.1063/1.5083186 


\title{
Photo-dissociation of naphthalene dimer cations stored in a compact electrostatic ion storage ring
}

Cite as: J. Chem. Phys. 150, 054303 (2019); doi: 10.1063/1.5055939

Submitted: 12 September 2018 - Accepted: 14 January 2019 •

Published Online: 7 February 2019

\section{J. Bernard, a) (iD) A. Al-Mogeeth, A.-R. Allouche, (D) L. Chen, G. Montagne, and S. Martin}

\author{
AFFILIATIONS \\ Institut Lumière Matière, UMR5306 Université Lyon 1-CNRS, Université de Lyon, Villeurbanne Cedex 69622, France
}

a) Author to whom correspondence should be addressed: jerome.bernard@univ-lyon1.fr

\begin{abstract}
Naphthalene dimer cations $\left[\mathrm{C}_{10} \mathrm{H}_{8}\right]_{2}{ }^{+}$have been produced by using an electron cyclotron resonance plasma ion source and stored in a compact electrostatic ion storage ring. We show that the radiative cooling of these cations is much slower than the isolated monomer naphthalene cations. We also report on photo-dissociation studies in the gas phase of naphthalene dimer cations at high internal energy. The dissociation energy is estimated to $0.5 \mathrm{eV}$ in close agreement with previous measurements but a factor of 2 smaller than recent (density functional theory (DFT) and ab initio) theoretical studies. As uncertainties on theory as well as on the experiment cannot be as large as this difference, we conclude that this discrepancy may be due to temperature effects with possible isomerization. As an interpretation of the photo-dissociation spectrum of naphthalene dimer cations, we propose a tentative simple analytical model based on effective Morse potentials. These effective potentials are expected to "average" temperature effects that would apparently result in a smaller energy difference between the fundamental and dissociation states due to the twisting vibration modes of the naphthalene dimer cations.
\end{abstract}

Published under license by AIP Publishing. https://doi.org/1 0.1063/1.5055939

\section{INTRODUCTION}

Numerous observational (as for instance recent observations with the Spitzer space telescope ${ }^{1-5}$ ), laboratory ${ }^{6-15}$ and theoretical ${ }^{16,17}$ studies on Polycyclic Aromatic Hydrocarbons (PAHs) have been motivated by the now commonly accepted presence of these large complex molecular systems in the interstellar medium. ${ }^{18,19}$ However, after a couple of decades of intensive research in this field, it has still not been possible to attribute any spectral feature, neither in absorption nor in emission, to a specific molecule of this family. Nonetheless, it has been estimated that the typical sizes of the PAHs are expected in the range of 50-150 carbon atoms. ${ }^{18}$ The key question of the growth of PAHs to such sizes is also a matter of debate. The first investigation by Frenklach and Feigelson suggested that most of the PAHs may be produced in the circumstellar shells of carbon-rich red giant stars. ${ }^{20}$ More recent studies suggest that small clusters of PAHs may form initial nuclei in the soot formation of hydrocarbon combustions and in the formation of carbonaceous particles in astrophysical environments. ${ }^{21}$ On the other hand, in PhotoDissociation Regions (PDRs) of nebulae, free flying PAHs are expected to result from the dissociation of very small carbonaceous grains (VSGs) under UV radiation. ${ }^{22,23}$ Possible candidates for these VSGs could be PAH clusters. This led Rapacioli et al. ${ }^{24}$ to study theoretically the formation and destruction of neutral PAH clusters in the PDRs. Their results show that these clusters should be evaporated faster than those reformed under a certain limit size. However, these authors also mention that cationic PAH clusters may be more stable than neutral ones. ${ }^{24}$

Small clusters of PAHs have been studied experimentally in neutral, ${ }^{25}$ anionic, ${ }^{26}$ and cationic forms and theoretically ${ }^{24}$ in the past two decades. The very first photo-dissociation spectra of naphthalene dimer cations were obtained in solution by Badger and Brocklehurst ${ }^{27}$ and in the gas phase by 
Inokuchi et al. ${ }^{28}$ The photo-absorption spectrum of naphthalene dimer cations exhibits two features: the most intense in the near infrared region is attributed to the charge resonance $(\mathrm{CR})$ transition, and the other one in the visible range is attributed to the local excitation (LE) transition. The dissociation energy leading to the separation into a neutral monomer and a charged monomer had then been roughly estimated as half of the CR transition energy as shown in the energy diagram of Fig. 1. Other methods have been used to measure the dissociation energy of the naphthalene dimer cations: thermodynamics coupled to mass spectrometry ${ }^{29}$ and two-color two-photon measurements of the ionization and appearance energies. ${ }^{30}$ Concerning the structure of the naphthalene dimer cations in the ground state, several theoretical papers $^{31}$ have shown that the sandwich parallel structure is favored. Ion mobility measurements have also confirmed this result. ${ }^{32}$ However, there is still some dispersion in the experimental measurements (up to $\pm 0.3 \mathrm{eV})^{27,29,30}$ as well as in the theoretical values of the dissociation energy of the naphthalene dimer cation (see Table I). The systematic difference between experimental and theoretical values of about a factor of two could hardly be attributed to uncertainties nor to too strong approximations in the calculations. It is noteworthy that the configurations of naphthalene dimer cations are very sensitive to the internal energy, leading to possible isomerization even at rather low internal energies. For instance, for pyrene dimer cations, Dontot et al. have shown that the potential energy curves (PECs) are expected to change significantly with the twisting angle between the two monomers (see Fig. 4 in Ref. 31).

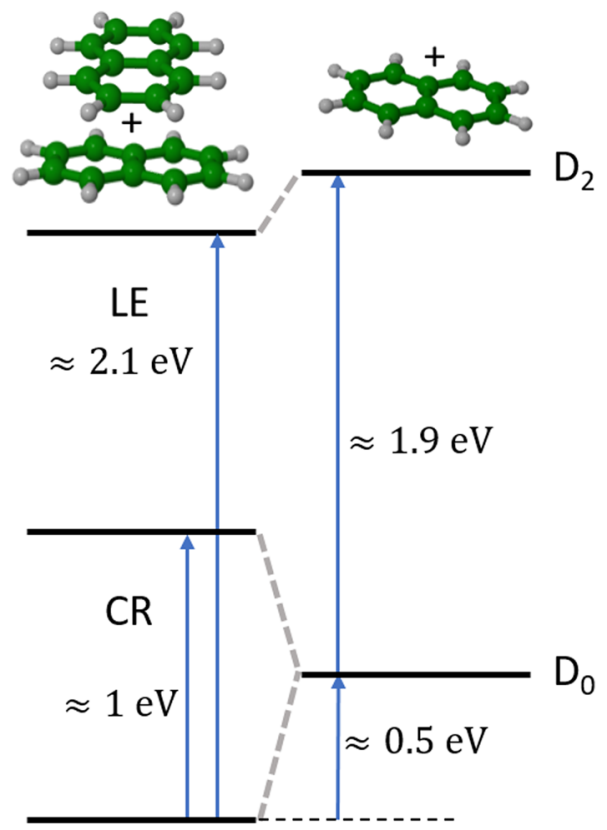

FIG. 1. Approximate diagram of electronic energy level of naphthalene and naphthalene dimer cations.
TABLE I. Theoretical (in sandwich configuration) ${ }^{31}$ and experimental dissociation energy for the ${ }^{2} \mathrm{~B}_{1 \mathrm{~g}}$ ground state of the naphthalene dimer.

\begin{tabular}{|c|c|c|}
\hline & Method (Ref.) & $\mathrm{D}_{\mathrm{e}}(\mathrm{eV})$ \\
\hline \multirow{4}{*}{ Theory } & DFTB-CI ${ }^{31}$ & 0.9 \\
\hline & $\mathrm{CAS}(1 / 1) \mathrm{PT} 2 / \mathrm{B1}^{31}$ & 1.17 \\
\hline & $\begin{array}{l}\text { Valence-band model } \\
\text { potential }^{58}\end{array}$ & 0.86 \\
\hline & $\begin{array}{l}\text { TPSS0/def2-qzvpp/D3BJ } \\
\text { (this work) }\end{array}$ & 1.18 \\
\hline \multirow{4}{*}{ Experiment } & $\begin{array}{l}\text { Absorption spectroscopy } \\
\text { (solution) }\end{array}$ & 0.59 \\
\hline & $\begin{array}{l}\text { Appearance energy } \\
\text { (gas phase) }\end{array}$ & $0.68 \pm 0.04$ \\
\hline & $\begin{array}{l}\text { Thermochemistry } \\
\text { (gas phase) }\end{array}$ & $0.77 \pm 0.03$ \\
\hline & $\begin{array}{l}\text { Photo-dissociation (gas phase) } \\
\text { (this work) }\end{array}$ & $0.5 \pm 0.1$ \\
\hline
\end{tabular}

Electrostatic storage rings and traps have now proved to be powerful devices to study the dynamics of molecular ions in the gas phase over a wide time range from microseconds to seconds at room temperatures or down to ultra-cold conditions. ${ }^{8,33-40}$ In this work, we used the Mini-Ring, ${ }^{41}$ a homedesigned electrostatic ion storage ring to store up to $100 \mathrm{~ms}$ ion beams in order to characterize the dynamics of dissociation and of radiative relaxation of stored naphthalene dimer cations and to record as well their dissociation spectrum. We have evidenced experimentally that prompt dissociation is only observed by giving an upper limit of the dissociation lifetime after laser absorption. We have also confirmed experimentally that dissociation can only occur via separation in two monomers using a coincidence detection. Finally, we propose a model for the recorded photo-dissociation spectrum using "effective" Morse potential energy curves that average the effects of the set of real isomer-dependent potential energy hypersurfaces.

\section{EXPERIMENT}

\section{A. Production and formation of naphthalene dimer cations in the ECR ion source}

electron cyclotron resonance (ECR) ion sources are widely employed in low and high energy accelerator facilities. ${ }^{42-44}$ They are well-known to produce high intensity beams of singly charged to highly charged ion beams $\left(\mathrm{Ar}^{18+}\right.$, $\left.\mathrm{Xe}^{30+}, \ldots\right)$. In short, a gas to be ionized is injected at low pressure (about $10^{-6}$ mbar) in a chamber surrounded by magnets providing strong magnetic fields for plasma confinement. A high frequency (HF) field is injected such that a resonance surface between the electron gyromagnetic movement and the HF is formed. At this resonance surface, electrons can be accelerated up to $\mathrm{keV}$ energies depending on several factors: amplitude of the HF, electron mean-free path, etc. We used a Nanogan I ECR ion source (purchased from the Pantechnik company), using optimized settings for the formation 
of a rather cold plasma in order to gently ionize molecules, i.e., in order to avoid dissociation in the source. The naphthalene, $\mathrm{C}_{10} \mathrm{H}_{8}$, powder was purchased from Fluka Chemicals (purity 99\%) and used as delivered. This powder was placed into a reservoir with a hole of $1 \mathrm{~mm}$ in diameter and connected to the ECR source via a valve whose opening could be controlled in order to optimize the naphthalene gas pressure in the source (we let the naphthalene powder sublimate under vacuum without any heating). The real pressure inside the source could not be directly measured. However, it is expected that the pressure measured by the vacuum gauge located in the next chamber of the beam line was rather proportional to the pressure in the source in the considered 1-30 $\times 10^{-6}$ mbar range (free molecular flow regime). Knowing the pumping speed and the conductance determined by the sizes of the tube and holes between the source and the next chamber (plasma electrode with a hole of $2 \mathrm{~mm}$ in diameter), we could estimate the pressure in the source to be typically in the range of $10^{-1}-10^{-2}$ mbar. The ECR ion source was biased to $12 \mathrm{kV}$ and connected to a beam line (via an insulating flange) at the ground potential in order to produce $12 \mathrm{keV}$ singly charged ion beams.

A $90^{\circ}$ magnet was used to select the naphthalene dimer cations. Mass spectra were recorded by scanning the selection magnet and by letting a continuous ion beam complete a single revolution in the Mini-Ring and recording the beam current collected by a Faraday cup (FC in Fig. 2). The optimization of the ECR source operation was made by recording mass spectra. Two typical mass spectra are shown in Fig. 3. With the combination of the selection magnet (velocity analysis) and the Mini-Ring used as an electrostatic energy analyzer (detection after one revolution), the mass resolution was improved (compared to the magnetic selection only) up to about $\mathrm{m} / \Delta \mathrm{m}=3000$. The mass spectrum of Fig. 3(a) shows only intact naphthalene dimer cations in the displayed mass range (and a peak resulting from dimers with one isotope ${ }^{13} \mathrm{C}$ ). In contrast, the mass spectrum of Fig. 3(b) shows mostly fragmented dimer cations, corresponding to the loss of one or several $\mathrm{H}$ atoms. These spectra have been recorded for two different naphthalene gas pressures in the ECR source. As the pressure could not be measured directly in the source, it would be rather difficult to model quantitatively the dependence of the plasma temperature on the naphthalene pressure. However, it may be qualitatively explained as follows. At high pressure, the mean free path of the electrons is rather short such that they cannot be much accelerated (by the electron cyclotron resonance effect) between two collisions. Consequently, most of the electrons of the plasma could not acquire sufficient kinetic energies to ionize and dissociate naphthalene neutral molecules. Hence, a high source pressure results in a low plasma temperature and no dissociated naphthalene dimer cations were observed. Oppositely, a lower pressure induced a higher electronic temperature and consequently the observation of dimers with missing hydrogen atoms. These observations also support the following formation mechanism of the dimer cations: naphthalene molecules were ionized and possibly dissociated by emission of one or several $\mathrm{H}$ atoms; then, those cation monomers may encounter another neutral and intact monomer in the plasma to form the dimer. There is a long distance attractive force between the cationic and the neutral monomers due to polarization leading to the formation of the dimer. We may exclude the case of intact ionized monomers sticking with fragmented neutral monomers because the latter are expected to be present in the plasma in much smaller quantities compared to the intact ones that are constantly injected in the plasma at room temperature.

\section{B. The Mini-Ring and neutral detection systems}

The Mini-Ring has been extensively described in Refs. 8 and 41. Briefly, it is composed of four parallel-plate electrostatic deflectors, labelled $\mathrm{D}_{1}-\mathrm{D}_{4}$ in the order they are encountered by the ion beam, and 2 conical shaped electrodes, labelled $\mathrm{C}_{1}$ and $\mathrm{C}_{2}$, used as electrostatic focusing mirrors (Fig. 2). Ion bunches of 1-5 $\mu$ s are prepared in the beam line between the selection magnet and the Mini-Ring by chopping the continuous beam with a homemade chopper composed of two parallel plates. In usual operation, dissociation events of the circulating molecular ions occurring in between

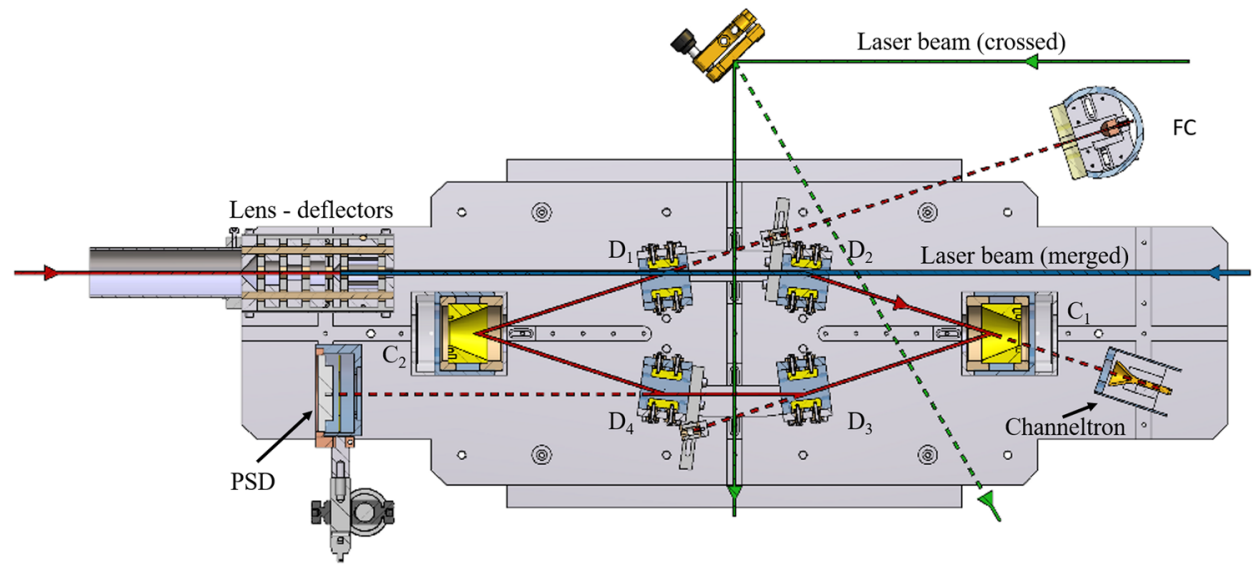

FIG. 2. Schematic view of the Mini-Ring, including detection systems (FC: Faraday cup, PSD: Position Sensitive Detector composed of microchannel plates and a resistive anode, and channeltron) and laser and ion pathways for merged beam (blue line) and crossed beam configurations (green solid line for recording photo-dissociation spectra and green dashed line for coincidence experiments). Deflectors $D_{i}$ and conical mirrors $C_{j}$ are numbered in the order they are encountered by the ion beam $(i=1-4, j=1,2)$. 


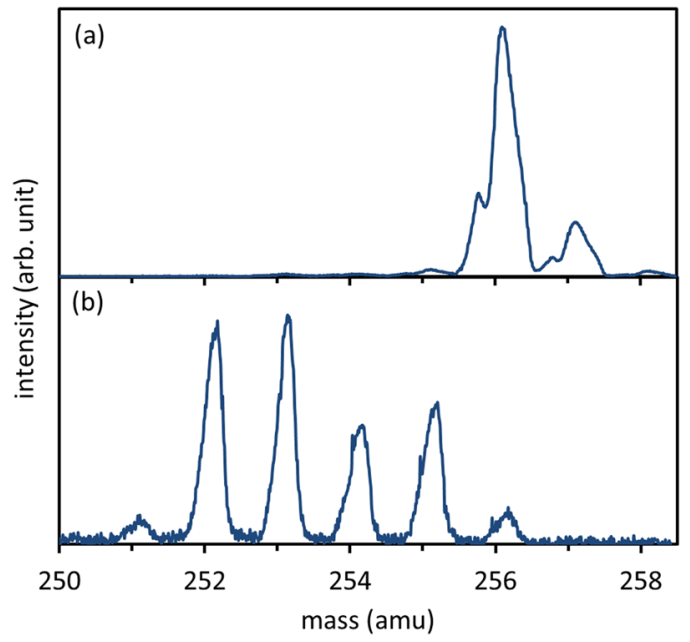

FIG. 3. Mass spectra (in the range 250-258 amu) recorded for a measured pressure of $2 \times 10^{-5} \mathrm{mbar}(\mathrm{a})$ and $5 \times 10^{-6} \mathrm{mbar}(\mathrm{b})$. Depending on the pressure, the temperature change of the plasma in the ECR ion source for these two pressures lead to the appearance of fragmented dimer cations.

the deflectors $\mathrm{D}_{3}$ and $\mathrm{D}_{4}$ can lead to neutral fragments that were detected by microchannel plates equipped with a resistive anode. This system provided time and position sensitive detection (PSD). The PSD was used for two purposes. First, optimized storage parameters were obtained with tests beams (e.g., $\mathrm{Ar}^{+}$) by reducing the spot size and the deviation of the center of the spot from turn to turn (i.e., reducing the amplitude of the betatron oscillations). Second, kinetic energy release (KER) could be estimated from the comparison of the spot size of the stored molecular ion beam with that of the test beam. $^{45}$

Recently, the Mini-Ring has been equipped with a channeltron at the extension of the straight line between $D_{2}$ and $\mathrm{C}_{1}$ for detecting the neutral fragments that could go through the hole of $\mathrm{C}_{1}$. Although not fully used in this work, the benefits brought by this detector are multiple: improved resolution of the decay time measurements, access to very short decay times (shorter than the half of a period) and coincidence experiments.

The vacuum chamber surrounding the Mini-Ring was equipped with several glass and quartz windows allowing for laser irradiation of the ion bunch parallel to the $D_{1}-D_{2}$ straight line in order to study laser-induced delayed dissociation and perpendicular to the ion bunch trajectory between $D_{3}$ and $\mathrm{D}_{4}$ in order to study prompt laser-induced dissociation. We used the wavelength-tunable (260-2000 nm) optical parametric oscillator (OPO) output of a 3 ns pulsed laser (EKSPLA model NT242) to record the photo-dissociation spectrum of the stored naphthalene dimer cations in the visible and near infrared range with photon energies from 0.5 to $5 \mathrm{eV}$.

\section{EXPERIMENTAL RESULTS}

A preliminary study with the naphthalene dimer cations was to confirm that the naphthalene dimer cations dissociate into two fragments of the same mass. To this purpose, we performed a coincidence experiment between neutrals detected by the channeltron and ions detected by the PSD. In this case, we used the Mini-Ring for half a revolution setting $C_{1}$ and $D_{3}$ voltages to half of their nominal values and $\mathrm{D}_{4}$ to $0 \mathrm{~V}$ to drive the cationic monomer fragment to the PSD. The laser beam (2nd harmonics of the Nd:YAG at $532 \mathrm{~nm}$ ) was deflected to cross the ion beam between $D_{2}$ and $C_{1}$ to enhance the number of dissociation events. The electronic signal from the channeltron was used as a start of a time-to-digital converter and the PSD as a stop. With these settings, we confirmed, as expected, that the naphthalene dimer cations dissociated into two fragments of equal masses. By scanning the voltages of $\mathrm{C}_{1}$ and $\mathrm{D}_{3}$, from $0 \mathrm{~V}$ to their nominal value $(2 \mathrm{kV}$ and $16 \mathrm{kV}$, respectively) we could not observe any other dissociation scheme for naphthalene dimer cations.

It is very instructive to study the "natural" decay due to the dissociation of molecular ions of high internal energy in a storage ring. ${ }^{46,47}$ The natural decay due to the dissociation of naphthalene dimer cations of high internal energy was recorded up to $100 \mathrm{~ms}$ storage time (Fig. 4). It could be fitted by two contributions: (i) a $\mathrm{t}^{-1}$ law, typical of a broad internal energy distribution and ${ }^{48}$ (ii) an exponential decay law, typical of dissociation due to collisions between the circulating ions and the residual gas (the residual pressure in the Mini-Ring vacuum chamber was of about $1 \times 10^{-9} \mathrm{mbar}$ ). This result differs from our previous studies on the anthracene monomer cation ${ }^{49}$ showing a $t^{-0.8}$ initial decay law and, after $1 \mathrm{~ms}$ of storage, a significantly steeper slope compared to this initial power decay. This steeper decay was attributed to the quenching of the dissociation process by a fast radiative cooling process due to fluorescence from thermally excited electronic states (also referred as Poincaré fluorescence ${ }^{50,51}$ or recurrent fluorescence ${ }^{34,35,52}$ ). Consequently, the present

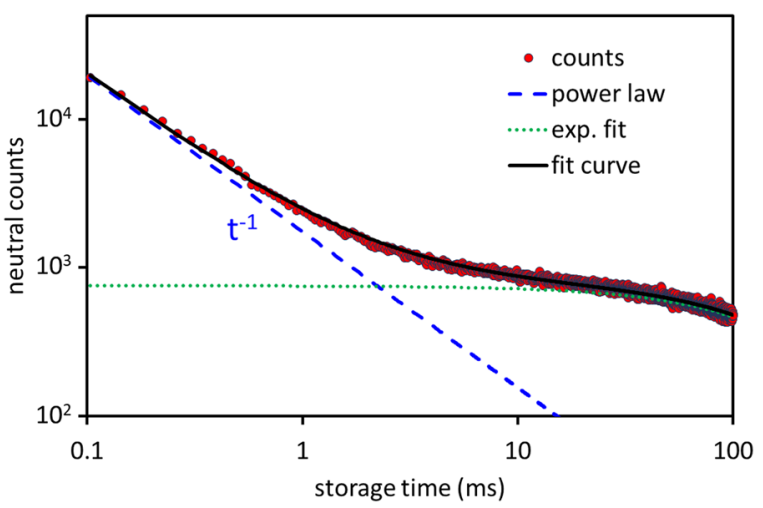

FIG. 4. Red dots: neutral counts on the PSD detector due to the natural decay of hot naphthalene dimer cations in the Mini-Ring and to collisions with the rest gas. The green dotted line is an exponential fit to neutral counts at long storage time accounting for neutrals resulting from collisions with the rest gas. The blue dashed curve is a $t^{-1}$ power law fit to the difference between the neutral and the exponential fit (considered as an estimation of the background at short storage times). 
$\mathrm{t}^{-1}$ decay law without any quenching shows that naphthalene dimer cations do not cool efficiently via a fast radiative cooling process such as the Poincaré fluorescence. However, vibrational infrared cooling may occur but on a much longer time scale compared to the Poincaré Fluorescence.

The absence of such Poincaré fluorescence is most probably due to the much smaller dissociation energy in the case of dimer cations (about $0.5 \mathrm{eV}$ ) compared to monomers (about $4.5 \mathrm{eV}$ ). Consequently, there is no energy matching between high vibrationally excited state of the ground electronic state and any low-lying bound electronic state that could fluoresce to the ground electronic state (see Fig. 1). Therefore, the population of any bound electronic excited states via the inverse internal conversion process, which is a necessary step for the fast radiative cooling via the Poincaré fluorescence, is highly improbable. Moreover, the first excited electronic state is dissociative. ${ }^{31}$ In case it can be thermally populated, dissociation only may occur. This is most probably the origin of the natural decay observed at short storage time.

Figure 5 displays a typical time spectrum of the neutral counts on the PSD for naphthalene dimer. The bunched structure (period of $7.92 \mu \mathrm{s}$ and width of about $1.5 \mu \mathrm{s}$ ) of the stored ions can be seen in the inset of Fig. 5. The narrow peak at $1833 \mu$ s corresponds to the huge enhancement of the neutral counts due to laser absorption (2nd harmonic-532 nm) in the crossed beam configuration in between $\mathrm{D}_{3}$ and $\mathrm{D}_{4}$. The laser power was set to about $80 \mu \mathrm{J} /$ pulse in order to avoid multiple photon absorption (measurement made downstream the whole laser pathway after the cross-beam region). This laser

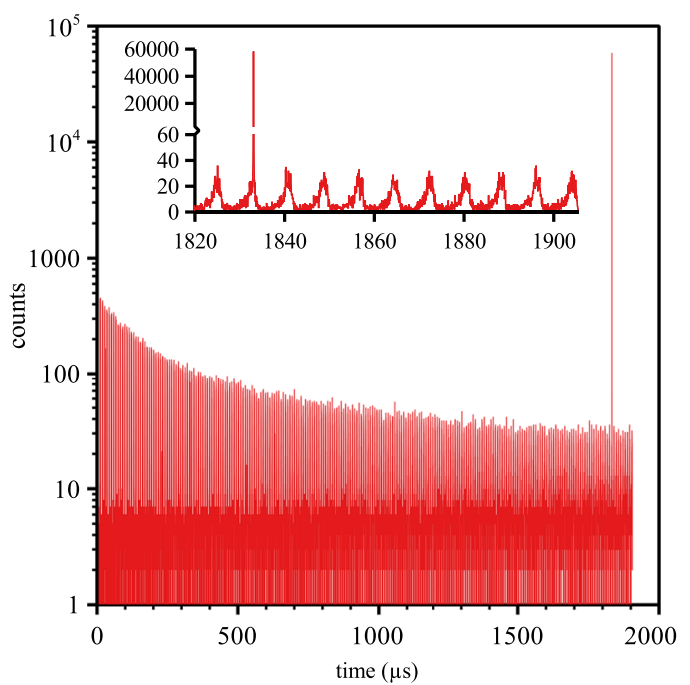

FIG. 5. Typical time of flight spectrum. The raw neutral counts were recorded as a function of the storage time. The peak at about $1833 \mu \mathrm{s}$ is due to the neutrals produced after laser absorption at $532 \mathrm{~nm}$ (second harmonic output of the $\mathrm{Nd}$ :YAG laser). This photo-dissociation signal was used to perform the photodissociation spectrum by scanning the laser wavelength. The inset shows a zoomin view around the photo-dissociation signal for better visualization of the bunched structure of the stored ions and of the signal to-noise ratio. pulse energy is of the same order of magnitude as the pulse energies in most of the visible range of the OPO laser that is used in the following. However, in the infrared range, the maximum OPO laser intensity is about one order of magnitude smaller than in the visible range. The storage time of about $2 \mathrm{~ms}$ prior laser irradiation has been chosen to reduce the contributions of the natural decay and of the collisions with residual gas (both considered as noise in the photo-dissociation spectrum) to the laser induced signal. Longer storage times would have the inconvenience to reduce the repetition rate of the experiment for a rather small gain on the signal to noise ratio. It is noteworthy that no delayed dissociation is observed on Fig. 5. It shows that the lifetime of the naphthalene dimer cations after absorption is much smaller than one of their revolution periods in the ring. Prompt dissociation only has been observed whatever the wavelength used in the visible and near infrared ranges. This absence of delayed dissociation suggests a non-statistical dissociation process for which the absorption in the CR band (near infrared range) and in the LE line (visible

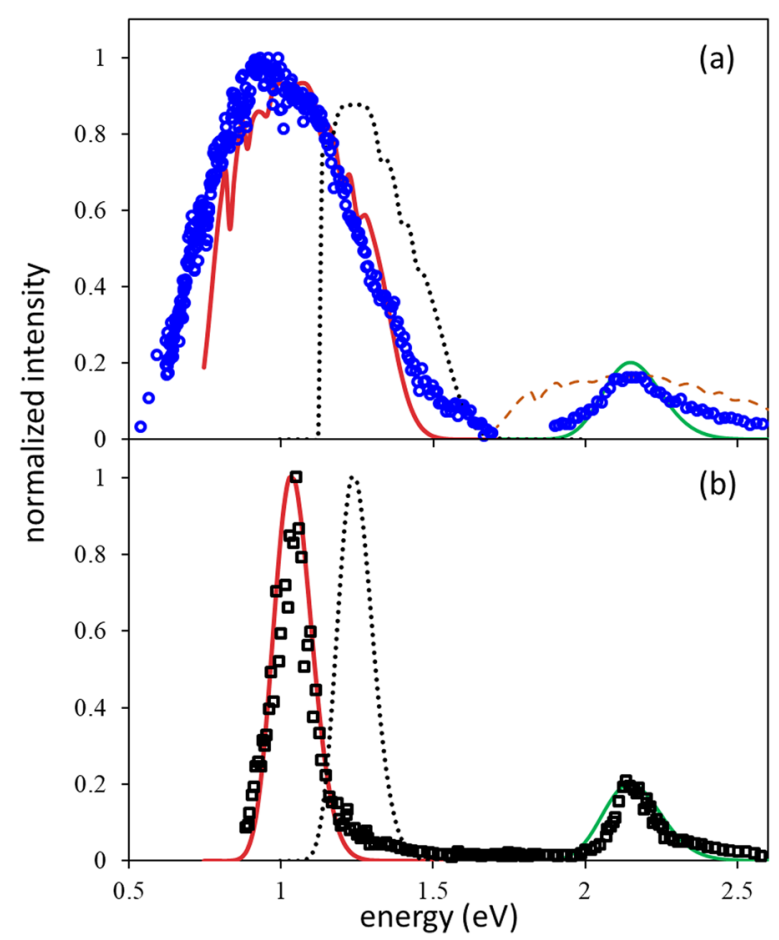

FIG. 6. (a) Experimental photo-dissociation spectrum (this work) obtained in hot conditions (blue circles). Simulated photo-dissociation spectra for the CR (red plain curve) and LE (orange dashed curve) including the contributions from v $=0$ to $v=4$ vibrational states calculated with effective Morse PEC parameters of Table III. (b) Experimental photo-dissociation spectrum (cold conditions) digitized from Ref. 28 (black squares). Simulated photo-absorption spectra for the CR (red plain curve) and LE (plain green line) using the $v=0$ vibrational state only. The LE simulated curve with $v=0$ (green plain line) of figure (b) has been also displayed in figure (a) for easy comparison. In (a) and (b), the dotted curves are the simulated CR lines calculated from the PECs of Ref. 31 for Boltzmann temperatures of $400 \mathrm{~K}$ and $10 \mathrm{~K}$, respectively. All the spectra have been normalized to 1. 
range) band would probably populate directly the dissociative excited states.

In order to record the photo-dissociation spectrum of the stored naphthalene dimer cations [Fig. 6(a)], the laser wavelength was scanned in the 400-690 nm and 800-2000 nm ranges (the laser intensity was too small in between these two ranges). An appropriate time gate was used to record the laser-induced neutral counts in order to reduce background counts due to collisions with the rest gas and the remaining natural decay. As expected, ${ }^{28}$ the photo-dissociation spectrum shows two broad peaks corresponding to the CR and LE bands centered at about $1 \mathrm{eV}$ and $2.2 \mathrm{eV}$. Hence, we estimate the dissociation energy to half of the CR transition energy, i.e., about $0.5 \mathrm{eV}$ in rather good agreement with other measurements (Table I). The CR band we have recorded is much broader than in the photo-dissociation spectrum of naphthalene dimer cations recorded by Inokuchi et al. ${ }^{28}$ [displayed in Fig. 6(b)]. This is most probably due to a much broader internal energy distribution of the stored naphthalene dimer cations in our experiment. Indeed, naphthalene dimer cations are produced in rather hot conditions in the plasma of the ECR ion source compared with a cold jet source as in the work of Inokuchi et al. ${ }^{28}$ Since there is no efficient radiative cooling for these species (see the above discussion about the natural decay), a $2 \mathrm{~ms}$ storage time is not long enough in order to have a significant change of the internal energy distribution. Another spectrum recorded after $20 \mathrm{~ms}$ storage time (not shown here), with less statistics though, did not show any significant change in the CR and LE peak widths. However, when comparing the spectra of Figs. 5(a) and 5(b), it is interesting to remark that the LE band is not much broadened in the hot conditions, as least not as much as the CR band.

\section{MODELLING AND DISCUSSION}

We have tentatively used the following strategy in order to test the PECs of Dontot et al. ${ }^{31}$ and to model the experimental photo-dissociation both for our study and the earlier one by Inokuchi et al::28

(i) Starting parameters were taken from PECs resulting from a fit with Morse potentials to $a b$ initio calculations of Dontot et al. ${ }^{31}$ The PECs along the inter-molecular separation of the 8 lowest electronic states of the naphthalene dimer cations in the sandwich configuration given in Ref. 31 could be nicely fitted by Morse potentials, assuming that the separation of the two monomers could be modelled in a similar way as for a diatomic molecule. Figure 7 displays the resulting fit for the ${ }^{2} \mathrm{~B}_{1 \mathrm{~g}}$ and ${ }^{2} \mathrm{~A}_{\mathrm{u}}$ states (CR transition). Very small differences between the Morse fit and the $a b$ initio calculation of Ref. 31 of the ${ }^{2} \mathrm{~B}_{1 \mathrm{~g}}$ and ${ }^{2} \mathrm{~A}_{\mathrm{u}}$ PECs can be barely seen only at rather large inter-monomer distance (greater than $5.5 \mathrm{~nm})$.

(ii) Morse PECs present the advantage of having analytical solutions to calculate the vibrational energy levels and eigenstates. ${ }^{53}$ Hence, the Franck-Condon factors for

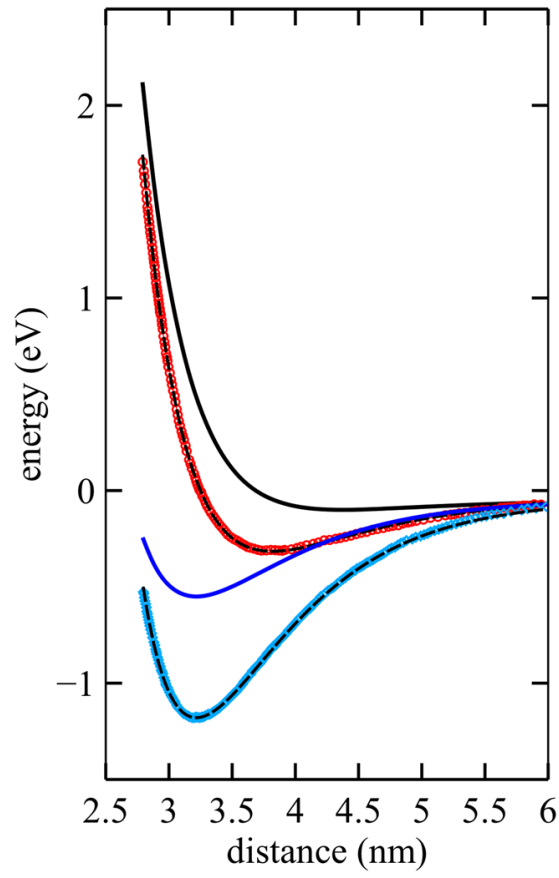

FIG. 7. PECs for the ${ }^{2} B_{1 g}$ and ${ }^{2} A_{u}$ states. Light blue diamonds $\left({ }^{2} B_{1 g}\right)$ and red circles $\left({ }^{2} A_{u}\right)$ : digitized from Ref. 31 . Black dashed lines: fits with Morse PECs on digitized data from Ref. 31 . Blue $\left({ }^{2} B_{1 g}\right)$ and black $\left({ }^{2} A_{u}\right)$ : modified Morse PECs used to model the CR line in Fig. 6.

the absorption transition to the ${ }^{2} \mathrm{~A}_{\mathrm{u}}$ dissociative state could be estimated following Ref. 54, p. 392 by the overlap of the wave functions obtained with parametrized PECs as shown in Fig. 8. As an example, the absorption line profile from $\mathrm{v}=0$ of the ${ }^{2} \mathrm{~B}_{1 \mathrm{~g}}$ ground state to the ${ }^{2} \mathrm{~A}_{\mathrm{u}}$ state is given by the red curve on the left side of Fig. 8.

(iii) We calculated the contributions of the five first vibrational levels $(v=0$ to $v=4)$ to the $\mathrm{CR}$ and the LE bands using Boltzmann distributions of the internal energy. The contribution of higher vibration levels has been neglected. These states may contribute mostly to the sides of the bands and might improve slightly the agreement between the model and experimental band widths.

Besides, we have calculated the energies of all the vibration modes of the naphthalene dimer cations using TPSS0 ${ }^{55}$ (Tao, Perdew, Staroverov, and Scuseria (TPSS) ${ }^{56}$ meta hybrid with $25 \%$ of exact exchange) density functional theory (DFT) functional with the def2-qzvpp basis set, including the dispersion via the D3 version of Grimme's approach with BeckeJohnson damping. ${ }^{57}$ The energies of the six lowest modes, corresponding to intermolecular motions between the two monomers, were found to be between 9.7 and $13.2 \mathrm{meV}$ (78.1 and $106.1 \mathrm{~cm}^{-1}$ ). They are in the same order of magnitude as the one calculated from the Morse potential used in the following, $h v_{0} \approx 7.8 \mathrm{meV}$, with the parameters of 


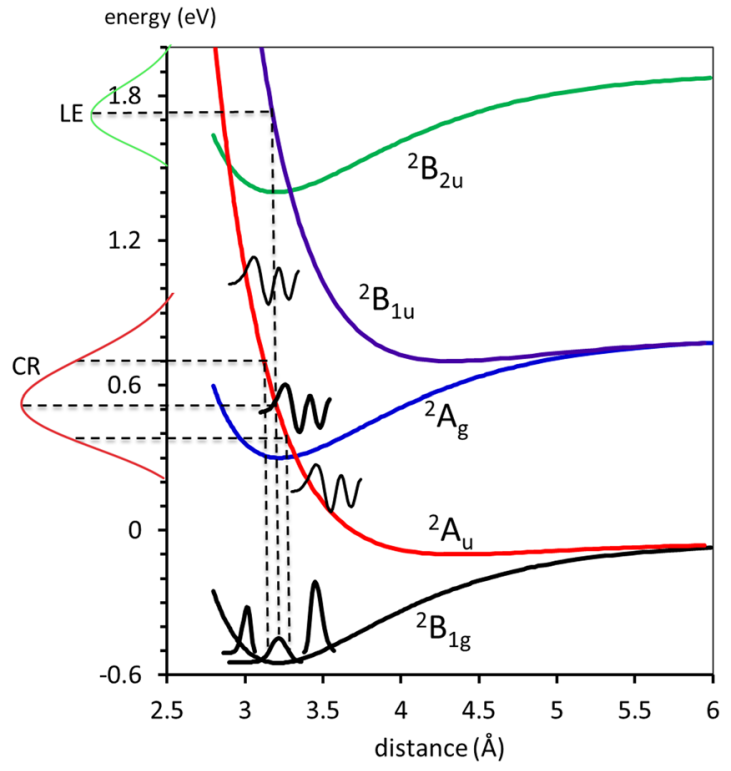

FIG. 8. Morse potential energy curves used in the calculations to simulate the photo-absorption spectra of Fig. 6 . For illustration purpose, the shape of the vibration wave functions for $v=0$ and $v=5$ of the electronic ground state ${ }^{2} B_{1 g}$, the shape of the wave function in the continuum of the ${ }^{2} \mathrm{~A}_{\mathrm{g}}$ dissociative state are displayed. On the left side of the vertical scale are shown the simulated absorption function for the CR and LE states (relative amplitudes of the CR and LE curves are arbitrary).

Table I for the ${ }^{2} \mathrm{~B}_{1 \mathrm{~g}}$ ground state. Intramolecular vibration energies were found at significantly higher energies (e.g., the lowest one was found at $23.2 \mathrm{meV}$ ). The rather small vibrational energy of the intermolecular motions shows that small internal energies may be sufficient to induce motions that are not accounted for in the one-coordinate PECs of Ref. 31.

Using directly the PECs resulting from the fit to those of Ref. 31, the $\mathrm{v}=0$ vertical absorption transition from the ground state, ${ }^{2} \mathrm{~B}_{1 \mathrm{~g}}$, to the first excited dissociative state, ${ }^{2} \mathrm{~A}_{\mathrm{u}}$ state, would correspond to a CR transition of about $1.23 \mathrm{eV}$ slightly larger than the experimental values $1.05 \mathrm{eV}$ in gas phase of Table II. Moreover, with these PECs, we could not model satisfactorily the CR band of both photo-dissociation spectra as shown by the dotted curves of Figs. 6(a) and 6(b). An optimization procedure of the parameters of the Morse potentials has been used in order to minimize the difference between the modeled and experimental CR bands. The resulting parameters of the Morse potentials are given in Table III

TABLE II. Energies of CR and LE transitions.

\begin{tabular}{llc}
\hline \hline & $\mathrm{CR}(\mathrm{eV})$ & $\mathrm{LE}(\mathrm{eV})$ \\
\hline Gas phase $^{28}$ & 1.05 & 2.17 \\
Liquid phase $^{59}$ & 1.21 & \\
Liquid phase $^{60}$ & 1.24 & 2.15 \\
This work & $1.0 \pm 0.2$ & \\
\hline
\end{tabular}

TABLE III. Parameters of the Morse potentials used in the model: dissociation energy $\left(D_{e}\right)$, equilibrium distance $\left(r_{e}\right)$, and minimum potential energy at $r_{e}\left(V_{e}\right)$.

\begin{tabular}{lcclll}
\hline \hline & ${ }^{2} \mathrm{~B}_{1 \mathrm{~g}}$ & ${ }^{2} \mathrm{~A}_{\mathrm{u}}$ & ${ }^{2} \mathrm{~B}_{1 \mathrm{u}}$ & ${ }^{2} \mathrm{~A}_{\mathrm{g}}$ & ${ }^{2} \mathrm{~B}_{2 \mathrm{u}}$ \\
\hline $\mathrm{D}_{\mathrm{e}}(\mathrm{eV})$ & 0.5 & 0.05 & 0.1 & 0.5 & 0.5 \\
$\mathrm{r}_{\mathrm{e}}(\AA)$ & 3.21 & 4.37 & 4.33 & 3.22 & 3.196 \\
$\mathrm{~V}_{\mathrm{e}}(\mathrm{eV})$ & -0.55 & -0.1 & 0.7 & 0.3 & 1.4 \\
\hline \hline
\end{tabular}

and the resulting PECs, displayed on Fig. 7, are rather different compared to those of Dontot et al. ${ }^{31}$

Including only the $\mathrm{v}=0$ vibrational state of the ${ }^{2} \mathrm{~B}_{1 \mathrm{~g}}$ ground state, the line profiles obtained with the adjusted Morse PECs fit nicely to experimental absorption curves obtained by Inokuchi et al. ${ }^{28}$ in cold conditions both for the CR and LE bands. This supports that it was possible to find a set of parameters for the ${ }^{2} \mathrm{~B}_{1 \mathrm{~g}}$ and ${ }^{2} \mathrm{~A}_{\mathrm{u}}$ states leading to PECs with optimized shapes around the equilibrium distance of the ground state. It is notable that the width of the model peak profile is very sensitive to the slope of the ${ }^{2} A_{u}$ dissociative state.

For higher vibration levels, the probability of presence is maximum at distances close to the edge of the potential well, as illustrated in Fig. 8 (see Ref. 54). Consequently, for the higher temperature conditions of our photo-dissociation spectrum [Figs. 6(a)], v > 0 levels mostly contribute to the sides of the line profiles. In order to reach a satisfactory model-experiment agreement, the high temperature spectrum gave more constraints in order to determine the shapes of the effective PECs of the ${ }^{2} \mathrm{~B}_{1 \mathrm{~g}}$ and $2 \mathrm{Au}$ states. We could reach a rather good agreement between the modeled and the experimental CR bands for our spectrum using a Boltzmann temperature of $400 \mathrm{~K}$. Using these effective potentials with a Boltzmann of $400 \mathrm{~K}$, the center of the modelled CR band is slightly shifted towards lower energy at $400 \mathrm{~K}$ compared to the low temperature spectrum. This shift estimated to about $-0.02 \mathrm{eV}$ is slightly smaller than the experimental shift but in the right direction though.

However, this shift due to the population of $v>0$ vibration level cannot explain the difference between the experimental CR lines and those obtained from the PECs from Dontot et al. ${ }^{31}$ The reason of this disagreement is most likely that these PECs are valid for motions in one coordinate only. As expected from our calculations of the vibration energies, rather small internal energies may lead to motions along other coordinates, such as, for instance, the aforementioned twisting motion calculated by Dontot et al. in the case of pyrene dimer cations. As shown in Fig. 4 of Ref. 31 for pyrene dimer cations, the gap between the ${ }^{2} \mathrm{~B}_{1 \mathrm{~g}}$ and ${ }^{2} \mathrm{~A}_{\mathrm{u}}$ states depends significantly on this twist angle such that the required energy for photo-dissociation is expected to be reduced with increasing twist angle up to $20^{\circ}$. Moreover, during the dissociation process, the relative motion between the two monomers may be rather complex involving other motions that are not included in the one-coordinate PECs. The estimation of the effect of several intermolecular (and possibly intramolecular) vibration motions would be needed in a full calculation. The Morse PECs resulting from 
the above adjustment procedure are expected to be "effective" potentials that average all temperature and possible isomerization effects such as the twisting angle between the two monomers.

The same method has been repeated for the LE absorption band. For the cold conditions of Fig. 6(b), the position and width of the model peak was found to be in good agreement with the experimental LE peak profile. However, for our spectrum [Fig. 6(a)], using the same temperature of $400 \mathrm{~K}$ as for the CR transition, the broadening of the model LE peak due to the inclusion of $\mathrm{v}>0$ states is about twice as broad as the experimental profile. In Fig. 6(a), we clearly see that the experimental LE band is not as broad as the CR band, but slightly broader than the LE band of the cold conditions as if, for the LE transition, the dimer had less vibrational energy. The diatomic model using effective Morse potentials for a single coordinate is possibly too crude to describe correctly the shape of the LE absorption peak profile. However, we may invoke two possible explanations for the small broadening of the LE peak at high temperature:

(i) It can be expected that the photon would be absorbed by one of the monomers. Therefore, the vibrational broadening would involve the intramolecular $\mathrm{C}-\mathrm{H}$ and $\mathrm{C}-\mathrm{C}$ vibrations of this monomer only. As these intramolecular vibrations have been calculated at much higher energy compared to the intermolecular vibrations, it can be expected that at the temperature of $400 \mathrm{~K}$ deduced from the CR broadening, the $\mathrm{v}>1 \mathrm{lev}-$ els of the intramolecular vibrations are very weakly populated.

(ii) The photo-absorption may not populate directly the dissociative state ${ }^{2} \mathrm{~B}_{1 \mathrm{u}}$, but the ${ }^{2} \mathrm{~B}_{2 \mathrm{u}}$ bound state. This state may undergo statistical dissociation via internal conversion leading to a thinner profile compared to the model the LE curve of Fig. 6(a). This tentative explanation is supported by the rather high oscillator strength of transitions at 2.1 and $2.2 \mathrm{eV}$ calculated by Dontot et al. ${ }^{31}$

More investigations would be needed to come to a more definitive conclusion about the evolution of the width of the LE band as a function of the temperature.

\section{CONCLUSION}

In summary, we have stored in the Mini-Ring beams of naphthalene dimer cations produced in an ECR ion source. Special tuning conditions, i.e., low HF power and high pressure, of this ion source have been discussed. Oppositely to monomer naphthalene cations, no fast reduction of the internal energy of the naphthalene dimer cations could be evidenced, demonstrating that the Poincaré (or recurrent) fluorescence is not an efficient process for the naphthalene dimer cations. The reason is that the low dissociation energy of the dimers do not allow any energy match between high vibrational states of the ground electronic state and the lower vibrational level of the first electronic excited state.
A photo-absorption spectrum of the naphthalene dimer cations has been recorded after $2 \mathrm{~ms}$ storage time in the Mini-Ring. CR and LE lines have been clearly identified as in previous studies in colder conditions. Effective Morse PECs for the ground and the first dissociative states have been used to compute a model photo-dissociation spectrum. It is expected that the proposed PECs average in some way the complexity induced by all the possible intermolecular motions. A more accurate modelling would require a considerable theoretical effort to sample the whole potential energy hypersurface in order to account for all possible vibration and rotation motions of all possible isomers. Nevertheless, additionally to the expected broadening, a shift towards lower energy of the CR absorption band was found with increasing temperature and was implicitly accounted for in the present modelling by choosing an appropriate parameter for the Morse PECs. However, this shift is not sufficient to explain the discrepancy between the experimental band centers and those obtained from the $a b$ initio PECs. We expect that, even at rather low temperatures, motions in all inter-molecular coordinates should be included as the corresponding vibration energies are rather small. More experimental and theoretical efforts would be needed in order to investigate thoroughly the temperature effects in $\mathrm{PAH}$ dimer cations.

\section{ACKNOWLEDGMENTS}

This work was supported by Grant No. ANR-10-BLAN-0426 of the ANR (Agence Nationale pour la Recherche). The authors would like to thank Christine Joblin and Mathias Rapacioli for valuable and intensive discussions concerning PAH dimers.

\section{REFERENCES}

${ }^{1}$ E. Peeters et al., "The rich 6 to $9 \mu \mathrm{m}$ spectrum of interstellar PAHs," Astron. Astrophys. 390(3), 1089-1113 (2002).

${ }^{2}$ E. Peeters, C. W. Bauschlicher, L. J. Allamandola, A. G. G. M. Tielens, A. Ricca, and M. G. Wolfire, "The PAH emission characteristics of the reflection nebula NGC 2023," Astrophys. J. 836(2), 198 (2017).

${ }^{3}$ D. J. Stock et al., "Polycyclic aromatic hydrocarbon emission in spitzer/IRS maps. I. Catalog and simple diagnostics," Astrophys. J. 819(1), 65 (2016).

${ }^{4}$ D. J. Stock and E. Peeters, "Polycyclic aromatic hydrocarbon emission in spitzer/IRS maps. II. A direct link between band profiles and the radiation field strength," Astrophys. J. 837(2), 129 (2017).

${ }^{\mathbf{5}}$ E. Peeters, "The infrared emission bands," Proc. Int. Astron. Union 9(S297), 187-196 (2013).

${ }^{6}$ A. Leger, L. D'Hendecourt, P. Boissel, and F. X. Desert, "Photo-thermodissociation. I. A general mechanism for destroying molecules," Astron. Astrophys. 213, 351-359 (1989).

${ }^{7}$ J. Montillaud, C. Joblin, and D. Toublanc, "Evolution of polycyclic aromatic hydrocarbons in photodissociation regions: Hydrogenation and charge states," Astron. Astrophys. 552, A15 (2013).

${ }^{8} \mathrm{~S}$. Martin et al., "Fast radiative cooling of anthracene observed in a compact electrostatic storage ring," Phys. Rev. Lett. 110(6), 063003 (2013).

${ }^{9}$ T. Giesen et al., "Molecular Spectroscopy," in Laboratory Astrochemistry, edited by S. Schlemmer, H. Mutschke, T. Giesen, and C. Jäger (Wiley-VCH Verlag GmbH \& Co. KGaA, 2014), pp. 13-108. 
${ }^{10} \mathrm{~J}$. Szczepanski, M. Vala, D. Talbi, O. Parisel, and Y. Ellinger, "Electronic and vibrational-spectra of matrix-isolated anthracene radical cationsExperimental and theoretical aspects," J. Chem. Phys. 98(6), 4494-4511 (1993).

${ }^{11}$ D. M. Hudgins, S. A. Sandford, and L. J. Allamandola, "Infrared spectroscopy of polycyclic aromatic hydrocarbon cations. 1. Matrix-isolated naphthalene and perdeuterated naphthalene," J. Phys. Chem. 98(16), 42434253 (1994)

${ }^{12}$ D. M. Hudgins and L. J. Allamandola, "Infrared spectroscopy of matrixisolated polycyclic aromatic hydrocarbon cations. 2 . The members of the thermodynamically most favorable series through coronene," J. Phys. Chem. 99(10), 3033-3046 (1995)

${ }^{13} \mathrm{~J}$. Oomens, A. G. G. M. Tielens, B. G. Sartakov, G. von Helden, and G. Meijer, "Laboratory infrared spectroscopy of cationic polycyclic aromatic hydrocarbon molecules," Astrophys. J. 591(2), 968 (2003).

${ }^{14}$ C. Joblin, P. Boissel, A. Leger, L. D'Hendecourt, and D. Defourneau, "Infrared spectroscopy of gas-phase PAH molecules. II. Role of the temperature," Astron. Astrophys. 299, 835 (1995).

${ }^{15}$ A. J. Huneycutt, R. N. Casaes, B. J. McCall, C.-Y. Chung, Y.-P. Lee, and R. J. Saykally, "Infrared cavity ringdown spectroscopy of jet-cooled polycyclic aromatic hydrocarbons," ChemPhysChem 5(3), 321-326 (2004).

${ }^{16} \mathrm{G}$. Malloci, C. Joblin, and G. Mulas, "On-line database of the spectral properties of polycyclic aromatic hydrocarbons," e-print arXiv:astro-Ph0701254 (2007).

${ }^{17}$ G. Malloci, G. Mulas, G. Cappellini, and C. Joblin, "Time-dependent density functional study of the electronic spectra of oligoacenes in the charge states $-1,0,+1$, and +2 ," Chem. Phys. 340(1-3), 43-58 (2007).

${ }^{18}$ A. G. G. M. Tielens, "The molecular universe," Rev. Mod. Phys. 85(3), 10211081 (2013)

${ }^{19} \mathrm{~A}$. Leger and J. L. Puget, "Identification of the 'unidentified' IR emission features of interstellar dust?," Astron. Astrophys. 137, L5-L8 (1984).

${ }^{20} \mathrm{M}$. Frenklach and E. D. Feigelson, "Formation of polycyclic aromatic hydrocarbons in circumstellar envelopes," Astrophys. J. 341, 372-384 (1989).

${ }^{21} \mathrm{H}$. Sabbah, L. Biennier, S. J. Klippenstein, I. R. Sims, and B. R. Rowe, "Exploring the role of PAHs in the formation of soot: Pyrene dimerization," J. Phys. Chem. Lett. 1(19), 2962-2967 (2010).

${ }^{22}$ M. Rapacioli, C. Joblin, and P. Boissel, "Spectroscopy of polycyclic aromatic hydrocarbons and very small grains in photodissociation regions," Astron. Astrophys. 429(1), 193-204 (2005).

${ }^{23}$ P. Pilleri, J. Montillaud, O. Berné, and C. Joblin, "Evaporating very small grains as tracers of the UV radiation field in photo-dissociation regions," Astron. Astrophys. 542, A69 (2012).

${ }^{24}$ M. Rapacioli, F. Calvo, C. Joblin, P. Parneix, D. Toublanc, and F. Spiegelman, "Formation and destruction of polycyclic aromatic hydrocarbon clusters in the interstellar medium," Astron. Astrophys. 460(2), 519-531 (2006)

${ }^{25} \mathrm{P}$. Benharash, M. J. Gleason, and P. M. Felker, "Rotational coherence spectroscopy and structure of naphthalene trimer," J. Phys. Chem. A 103(11), 1442-1446 (1999).

${ }^{26}$ J. K. Song, N. K. Lee, J. H. Kim, S. Y. Han, and S. K. Kim, "Anion clusters of anthracene, $\mathrm{An}_{n}{ }^{-}$(n=1-16)," J. Chem. Phys. 119(6), 3071-3077 (2003).

${ }^{27} \mathrm{~B}$. Badger and B. Brocklehurst, "Formation of dimer cations of aromatic hydrocarbons," Nature 219(5151), 263 (1968).

${ }^{28}$ Y. Inokuchi, K. Ohashi, M. Matsumoto, and N. Nishi, "Photodissociation spectrum of naphthalene dimer cation," J. Phys. Chem. 99(11), 3416-3418 (1995).

${ }^{29} \mathrm{M}$. Meot-Ner, "Dimer cations of polycyclic aromatics. Experimental bonding energies and resonance stabilization," J. Phys. Chem. 84(21), 2724-2728 (1980).

${ }^{30}$ T. Fujiwara and E. C. Lim, "Binding energies of the neutral and ionic clusters of naphthalene in their ground electronic states," J. Phys. Chem. A 107(22), 4381-4386 (2003).

${ }^{31}$ L. Dontot, N. Suaud, M. Rapacioli, and F. Spiegelman, "An extended DFTBCI model for charge-transfer excited states in cationic molecular clusters: Model studies versus $a b$ initio calculations in small PAH clusters," Phys. Chem. Chem. Phys. 18(5), 3545-3557 (2016).
${ }^{32} \mathrm{~T}$. Beitz, R. Laudien, H.-G. Löhmannsröben, and B. Kallies, "Ion mobility spectrometric investigation of aromatic cations in the gas phase," J. Phys. Chem. A 110(10), 3514-3520 (2006).

${ }^{33} \mathrm{H}$. T. Schmidt et al., "First storage of ion beams in the double electrostatic ion-ring experiment: DESIREE," Rev. Sci. Instrum. 84(5), 055115 (2013).

${ }^{34} \mathrm{~V}$. Chandrasekaran et al., "Determination of absolute recurrent fluorescence rate coefficients for $\mathrm{C}_{6}{ }^{-}$," J. Phys. Chem. Lett. 5(23), 4078-4082 (2014).

${ }^{35} \mathrm{G}$. Ito et al., "Cooling dynamics of photoexcited $\mathrm{C}_{6}{ }^{-}$and $\mathrm{C}_{6} \mathrm{H}^{-}$," Phys. Rev. Lett. 112(18), 183001 (2014)

${ }^{36}$ S. P. Møller, "ELISA, and electrostatic storage ring for atomic physics," Nucl. Instrum. Methods Phys. Res., Sect. A 394(3), 281-286 (1997).

${ }^{37}$ D. Zajfman et al., "Electrostatic bottle for long-time storage of fast ion beams," Phys. Rev. A 55(3), R1577-R1580 (1997).

${ }^{38}$ R. von Hahn et al., "The cryogenic storage ring CSR," Rev. Sci. Instrum. 87(6), 063115 (2016).

${ }^{39} \mathrm{C}$. R. Calvert et al., "LIAD-fs scheme for studies of ultrafast laser interactions with gas phase biomolecules," Phys. Chem. Chem. Phys. 14(18), 6289-6297 (2012).

${ }^{40}$ T. Doussineau, R. Antoine, M. Santacreu, and P. Dugourd, "Pushing the limit of infrared multiphoton dissociation to megadalton-size DNA ions," J. Phys. Chem. Lett. 3(16), 2141-2145 (2012).

${ }^{41} \mathrm{~J}$. Bernard et al., "A 'tabletop' electrostatic ion storage ring: Mini-ring," Rev. Sci. Instrum. 79(7), 075109 (2008).

${ }^{42}$ R. Geller, Electron Cyclotron Resonance Ion Sources and ECR Plasmas (CRC Press, 1996)

${ }^{43}$ R. Geller, "ECRIS: The electron-cyclotron resonance ion sources," Annu. Rev. Nucl. Part. Sci. 40, 15-43 (1990).

${ }^{44} \mathrm{~L}$. Maunoury et al., "LIMBE: A new facility for low energy beams," Rev. Sci. Instrum. 73(2), 561-563 (2002).

${ }^{45} \mathrm{M}$. Ji, G. Montagne, R. Bredy, J. Bernard, L. Chen, and S. Martin, "Ion beam characterization in the mini-ring and kinetic energy release measurement of unimolecular decay of anthracene cations," Phys. Scr. T156, 014092 (2013).

${ }^{46} \mathrm{~L}$. H. Andersen, "Thermionic electron emission from $\mathrm{SF}_{6}{ }^{-}$," Phys. Rev. A 78(3), 032512 (2008).

${ }^{47} \mathrm{~J}$. U. Andersen et al., "Power-law decay of collisionally excited amino acids and quenching by radiative cooling," Eur. Phys. J. D 25(2), 139-148 (2003).

${ }^{48}$ K. Hansen, J. U. Andersen, P. Hvelplund, S. P. Møller, U. V. Pedersen, and V. V. Petrunin, "Observation of a 1/t decay law for hot clusters and molecules in a storage ring," Phys. Rev. Lett. 87(12), 123401 (2001).

${ }^{49} \mathrm{~J}$. Bernard et al., "Cooling of PAH cations studied with an electrostatic storage ring," Nucl. Instrum. Methods Phys. Res., Sect. B 408, 21-26 (2017).

${ }^{50} \mathrm{~A}$. Léger, P. Boissel, and L. d'Hendecourt, "Predicted fluorescence mechanism in highly isolated molecules: The Poincaré fluorescence," Phys. Rev. Lett. 60(10), 921-924 (1988).

${ }^{51}$ P. Boissel, P. de Parseval, P. Marty, and G. Lefevre, "Fragmentation of isolated ions by multiple photon absorption: A quantitative study," J. Chem. Phys. 106(12), 4973-4984 (1997).

${ }^{52}$ Y. Ebara et al., "Detection of recurrent fluorescence photons," Phys. Rev. Lett. 117(13), 133004 (2016).

${ }^{53} \mathrm{P} . \mathrm{M}$. Morse, "Diatomic molecules according to the wave mechanics. II. Vibrational levels," Phys. Rev. 34(1), 57-64 (1929).

${ }^{54}$ G. Herzberg, Molecular Spectra (D. Van Nostrand Company, Inc., 1950), Vol. I.

${ }^{55}$ S. Grimme, J. Antony, S. Ehrlich, and H. Krieg, "A consistent and accurate $a b$ initio parametrization of density functional dispersion correction (DFT-D) for the 94 elements H-Pu," J. Chem. Phys. 132(15), 154104 (2010).

56J. Tao, J. P. Perdew, V. N. Staroverov, and G. E. Scuseria, "Climbing the density functional ladder: Nonempirical meta-generalized gradient approximation designed for molecules and solids," Phys. Rev. Lett. 91(14), 146401 (2003). 
${ }^{57}$ S. Grimme, S. Ehrlich, and L. Goerigk, "Effect of the damping function in dispersion corrected density functional theory," J. Comput. Chem. 32(7), 1456-1465 (2011).

${ }^{58}$ B. Bouvier, V. Brenner, P. Millié, and J.-M. Soudan, "A model potential approach to charge resonance phenomena in aromatic cluster ions," J. Phys. Chem. A 106(43), 10326-10341 (2002).
${ }^{59} \mathrm{~T}$. N. Das, "Monomer and dimer radical cations of benzene, toluene, and naphthalene," J. Phys. Chem. A 113(23), 6489-6493 (2009).

${ }^{60} \mathrm{X}$. Cai, S. Tojo, M. Fujitsuka, and T. Majima, "Photodissociation of naphthalene dimer radical cation during the two-color two-laser flash photolysis and pulse radiolysis-laser flash photolysis," J. Phys. Chem. A 110(30), 9319-9324 (2006). 